Sear chi ng for O X-B mode- conver si on wi ndow wi th monitoring of st $r$ ay microwave radi at $i$ on in LHD

\begin{tabular}{|l|l|}
\hline $\begin{array}{l}\text { jour nal or } \\
\text { publ i cat i on } \mathrm{titl} \text { e }\end{array}$ & Revi ew of Sci ent i f i c I nst r ument s \\
\hline vol une & Vol . 77 \\
\hline number & I ssue 10 \\
\hline page range & pp. 10E931- 1 10E931- 3 \\
\hline year & 2006-10-01 \\
\hline URL & ht t p: //hdl . handl e. net /10655/3870 \\
\hline
\end{tabular}




\title{
Searching for $O-X-B$ mode-conversion window with monitoring of stray microwave radiation in LHD
}

\author{
H. Igami and S. Kubo \\ National Institute for Fusion Science, 322-6 Oroshi-cho, Toki, Gifu 509-5292, Japan \\ H. P. Laqua \\ Max-Plank-Institut für Plasmaphysik, EURATOM Association, D-17491 Greifswald, \\ Federal Republic of Germany \\ K. Nagasaki \\ Institute of Advanced Energy, Kyoto University, Gokasyo, Uji, Kyoto 611-0011, Japan \\ S. Inagaki, T. Notake, T. Shimozuma, Y. Yoshimura, \\ T. Mutoh, and LHD Experimental Group \\ National Institute for Fusion Science, 322-6 Oroshi-cho, Toki, Gifu 509-5292, Japan
}

(Received 9 May 2006; presented on 9 May 2006; accepted 6 July 2006; published online 23 October 2006)

In the Large Helical Device, the stray microwave radiation is monitored by using so-called sniffer probes during electron cyclotron heating. In monitoring the stray radiation, we changed the microwave beam injection angle and search the $O-X-B$ mode-conversion window to excite electron Bernstein waves (EBWs). When the microwave beam is injected toward the vicinity of the predicted $O-X-B$ mode-conversion window, the electron temperature rises in the central part of overdense plasmas. In that case, the stray radiation level near the injection antenna becomes low. These results indicate that monitoring the stray radiation near the injection antenna is helpful in confirming the effectiveness of excitation of EBWs simply without precise analysis. () 2006 American Institute of Physics. [DOI: 10.1063/1.2336460]

\section{INTRODUCTION}

Monitoring of the stray radiation with so-called sniffer probes ${ }^{1}$ is helpful in confirming the effectiveness of electron cyclotron heating $(\mathrm{ECH})$ simply. If the level of the stray radiation is low, we can guess that the injected microwave power is absorbed somewhere in the vacuum chamber, i.e., in the plasma, on the vacuum vessel wall, or on the surface of inner equipment. If electron Bernstein waves (EBWs) are successfully excited by microwave injection and absorbed in the plasma, the level of the stray radiation may decrease. When the superimposed microwave power is very low, it is difficult to find out the increase of stored energy and local increase of electron temperature profile of Thomson scattering measurement. Therefore it is helpful to monitor the stray radiation signals in trial heating experiments.

In some high performance plasmas of fusion-oriented devises, the electron plasma frequency $\omega_{p}$ exceeds the electron cyclotron frequency $\Omega$ around the electron cyclotron resonance (ECR) layer. In such "overdense" plasmas, normal propagation modes of electromagnetic waves cannot access the ECR layer, thus the usual way of ECH by normal ordinary $(O-)$ and extraordinary $(X-)$ modes is not available. ECH by electrostatic EBWs has been expected for ECH in high density plasmas for decades. ${ }^{2-7}$ EBWs can propagate without density limit and are absorbed in the ECR layer. They are required to be excited via mode-conversion process from electromagnetic waves. Thus it is necessary to inject microwaves even for the case of EBW-ECH. One way to excite EBWs is to inject $O$-mode waves from the lower mag- netic field side. If $O$-mode waves are injected at an appropriate angle, they can connect with $X$-mode waves near the plasma cutoff. The $X$-mode waves propagate toward the upper hylorid resonance (UHR) layer and are completely mode converted to EBWs. This so-called $O-X-B$ scheme is effective when the length scale of the density gradient $L_{n}=n_{e} /\left(d n_{e} / d x\right)$ is as long as $L_{n} / \lambda_{0} \geqslant 1,{ }^{6}$ where $n_{e}$ is the electron density and $\lambda_{0}$ is the vacuum wavelength of the incident microwaves.

On LHD, we have tried to excite EBWs with the $O-X-B$ scheme using the existing ECH system for $84 \mathrm{GHz}$ range of fundamental frequency. We changed the injection angle shot by shot to search the appropriate angle to excite EBWs via $O-X-B$ mode conversion $(O-X-B$ modeconversion window) with monitoring of the level of stray radiation. The EBW-ECH experiments based on this idea were already demonstrated in the TCV tokamak. ${ }^{7}$ In their experiments, stray radiation signals at different points showed similar tendencies when the injection angle was changed. However, in our experiments, stray radiation signals at different points showed different tendencies. The construction of this article is as follows: In Sec. II, the experimental setup is introduced, then the experimental results and discussions are presented in Sec. III. The conclusion is summarized in Sec. IV.

\section{EXPERIMENTAL SETUP}

A sniffer probe consists of a stainless steel oversized waveguide antenna coupled to a spherical copper cavity. The 


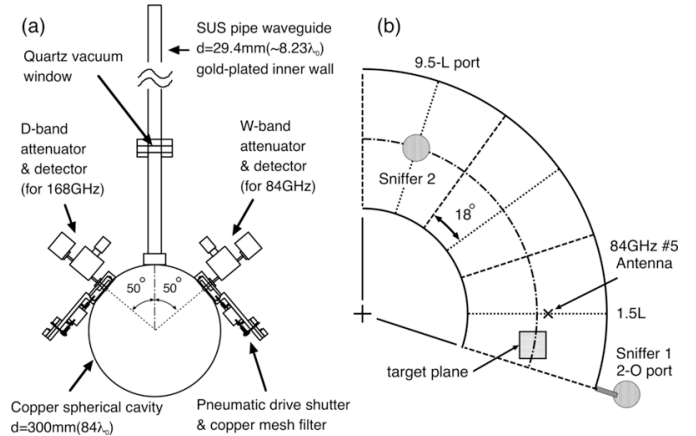

FIG. 1. (a) Schematic view of the sniffer probe installed in LHD. (b) Positional relationship between the microwave beam injection antenna and the sniffer probes.

diameter of the waveguide is several times of the vacuum wavelength, allowing many modes to propagate. All incoming waves into the integrating cavity are nearly equally coupled to the detector after several reflections. ${ }^{1}$ In Fig. 1(a), a schematic view of the sniffer probe installed in LHD is shown. A standard $W$-band microwave detector is connected to the cavity with an attenuator to pick up the stray radiation of $84 \mathrm{GHz}$ range used for fundamental resonance heating. A $D$-band microwave detector with an attenuator is also connected for $168 \mathrm{GHz}$ range for second harmonics heating. Two sniffer probes are installed in LHD as shown in Fig. 1 (b). One is installed in a horizontal port (2-O) and the other is installed in a bottom port (9.5-L). The distance from the main part of the vacuum chamber to the edge of the waveguide is $1150 \mathrm{~mm}$ at the 2-O port sniffer probe. That distance is $50 \mathrm{~mm}$ at the 9.5 -L port sniffer probe. Since 82.7 and $168 \mathrm{GHz}$ microwave injection antennas are installed just above the 9.5-U port, residual microwave power which does not suffer from the single-path absorption is directly irradiated around the antenna edge of the sniffer probe. Thus the protection against high power is needed for the sniffer probe at the $9.5-\mathrm{L}$ port. Pneumatically driven shutters controlled outside the experimental hall are mounted as shown in Fig. 1(a). When the shutter opens, copper meshed filters (40 mesh, $\phi 0.25 \mathrm{~mm}$, aperture of $15.5 \%$ ) are inserted in front of the attenuators. The attenuation is $\sim 20 \mathrm{~dB}$ for $168 \mathrm{GHz}$. In our $O-X-B$ EBW-ECH experiment, one of the $84 \mathrm{GHz}$ microwave injection antennas (Line No. 5) installed in the 1.5-L port was used. The microwave beams were injected toward the focal point on the midplane. Thus the focal point identifies the injection angle. We changed the focal point within the "target area," as shown in Fig. 1(b).

The $O-X-B$ mode-conversion rate $T$ is given as follows: ${ }^{3}$

$T=\exp \left\{-\pi \frac{\omega}{c} L_{n} \sqrt{\frac{Y}{2}}\left[2(1+Y)\left(N_{z}-N_{z, \mathrm{opt}}\right)^{2}+N_{y}^{2}\right]\right\}$,

where $Y=\Omega / \omega$ and $N_{z, \text { opt }}=\sqrt{Y /(1+Y)}$. Here we assume that the density increases along the $x$ direction and the external magnetic field $\mathbf{B}$ is paralell to the $z$ direction. $N_{y}$ and $N_{z}$ are the $y$ and $z$ components of the refractive index. In Fig. 2(a), a calculated $O-X-B$ mode-conversion rate is shown as a contour plot versus the radial and toroidal focal points $\left(R_{\text {focal }}\right.$, $\left.T_{\text {focal }}\right)$ within the target area, where $T_{\text {focal }}$ is the toroidal distance of the focal point from the 1.5 -L port and $R_{\text {focal }}$ is the
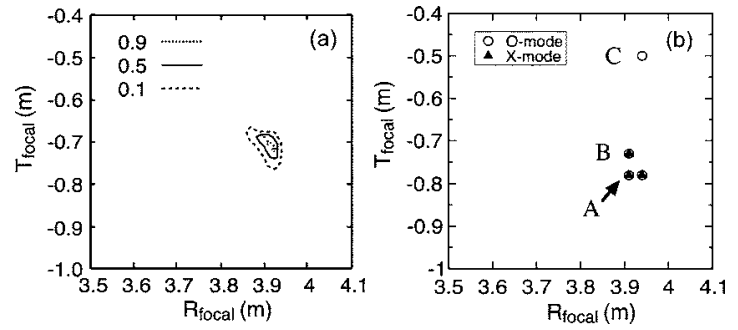

FIG. 2. (a) Calculated $O-X-B$ mode-conversion rate plotted vs the beam focal point. (b) Focal points on the midplane set in each experimental shot.

radial distance of the focal points from the center of the torus. In the calculation, we assumed that the edge of the electron density profile is located at $\rho=1.1$ and the plasma cutoff is located at $\rho=0.7$ in a magnetic configuration $R_{\text {axis }}=3.6 \mathrm{~m}$ and $B_{\text {axis }}=2.75 \mathrm{~T}$, where $\rho$ is the normalized minor radius. We can see that the width of the calculated "mode conversion window" is very narrow. The widths of toroidal and radial incident angles where the mode-conversion rate is larger than $50 \%$ are $\sim 2^{\circ}$ and $1^{\circ}$, respectively.

\section{EXPERIMENTAL RESULTS AND DISCUSSION}

In the experiment, $270 \mathrm{~kW}$ microwaves were injected with $100 \%$ power modulation of $47 \mathrm{~Hz}$ into the target plasma sustained by $5.1 \mathrm{MW}$ neutral beam injection (NBI). The magnetic configuration was $R_{\text {axis }}=3.6 \mathrm{~m}$ and $B_{\text {axis }}=2.75 \mathrm{~T}$. Nearly $O$-mode (left handed circular polarized) and $X$-mode (right handed circular polarized) waves were injected. The line averaged electron density at the central code exceeded the cutoff density of $84 \mathrm{GHz}, n_{c}=8.98 \times 10^{19} \mathrm{~m}^{-3}$ during superimposed injection. The reconstructed electron density profile from the line density profile indicates that the edge of the electron density profile is located at $\rho \sim 1.1$ and the plasma cutoff is located at $\rho \sim 0.7$. We changed the focal point shot by shot as shown in Fig. 2(b).

In Figs. 3(a)-3(d), the rise of electron temperature during modulated power injection of $47 \mathrm{~Hz}$ is plotted versus $\rho$. These results are obtained from fast Fourier transform (FFT) analysis of the electron cyclotron emission electron cyclotron emission (ECE) signals. The electron density did not exceed the cutoff density of the frequencies used in the ECE measurement in this experiment. Focal points $A, B$, and $C$ correspond to the focal points $A, B$, and $C$ marked in Fig. 2(b). As shown in Fig. 3(a), power absorption takes place when $O$-mode wave is injected toward the focal point $A$. When $O$-mode wave is injected to the focal point $B$, weaker power absorption takes place as shown in Fig. 3(b). As shown in Fig. 3(c), no power absorption takes place in the case of injection to the focal point $C$. In the case of $X$-mode wave injection to the focal point $A$, it seems that very weak absorption takes place as shown in Fig. 3(d).

In Figs. 4(a) and 4(d), stray radiation levels of the overdense plasmas at each probe are plotted as a function of the toroidal focal point. Note that both cases of $R_{\text {focal }}=3.91$, and $3.94 \mathrm{~m}$ are included at $T_{\text {focal }}=-0.78 \mathrm{~m}$. The signal level of the stray radiation at the 2-O sniffer probe is minimum at the focal point location $A \quad\left(R_{\text {focal }}, T_{\text {focal }}\right)=(3.91 \mathrm{~m},-0.78 \mathrm{~m})$. 

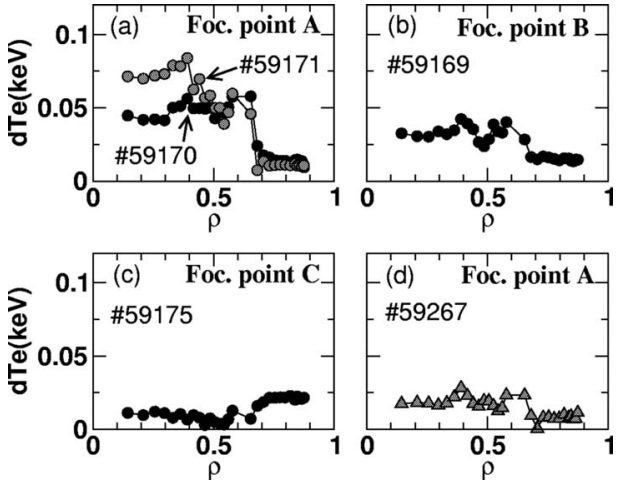

FIG. 3. [(a)-(c)] Electron temperature rise in the case of $O$-mode wave injection toward the focal points $A, B$, and $C$. (d) Electron temperature rise in the case of $X$-mode wave injection toward the focal point $A$. Focal points $A, B$, and $C$ correspond to those in Fig. 2(b).

When the microwave beam was injected toward the focal point $A$, the stray radiation level at 2-O sniffer probe became the minimum, while the stray radiation level at the $9.5-\mathrm{L}$ sniffer probe did not vary significantly when changing the focal point. There was no difference in the signal level between $O$-mode and $X$-mode wave injections. In the experiment, the overdense plasma often collapses during the discharge. In Figs. 4(b) and 4(e), the stray radiation levels when microwaves were injected into "collapsing" plasmas are plotted. In Figs. 4(c) and 4(f), the stray radiation levels when microwaves were injected into the vacuum space without plasma (after collapsing) were also plotted. At the 2-O sniffer probe, the stray radiation level was high when microwaves were injected into the overdense plasma toward the focal point $C$ and when they were injected into the collapsing plasmas. On the contrary, at 9.5-L sniffer probe, the stray radiation level was high when microwaves were injected into the vacuum space without a plasma. The stray radiation level when the microwaves were injected into the collapsing plasmas is as low as that in the case of injection into the overdense plasmas.

These tendencies can be explained as follows. As shown in Fig. 1(b), the incident microwave beam passes close to the 2-O port. If the plasma is not in the vacuum chamber, the beam hits the wall far from the $2-\mathrm{O}$ port. Then the microwaves are reflected to various directions and the power is gradually absorbed on the wall at every reflection. Therefore microwaves picked up at the 2-O sniffer probe are the integration of residual microwaves through multireflection in the case of vacuum injection. When an overdense plasma exists stably or is collapsing in the vacuum chamber, the incident microwave beam is reflected at the cutoff point located near the 2-O port, then the large portion of the reflected power almost directly comes back around the 2-O port, except for the case of microwave beam injection toward the modeconversion window. In that case, the microwaves are not reflected and come back around the $2-\mathrm{O}$ port. The microwave beam is injected in the direction opposite to the direction from the injection antenna to the $9.5 \mathrm{~L}$ sniffer probe as shown in Fig. 1(b). Microwaves picked up at the 9.5-L sniffer probe are the integration of residual microwaves through multireflection. When a plasma exists in the vacuum chamber, the power of microwaves is gradually absorbed every time the

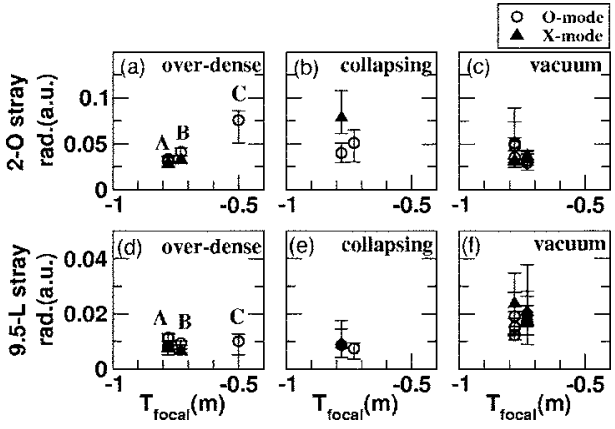

FIG. 4. Signal level of the stray radiation at 2-O and 9.5-L sniffer probes, for each case of injection into overdense plasmas, collapsing plasmas, and vacuum space.

waves are reflected on the wall and pass through the plasma. Even if the waves do not suffer the single-path absorption, a large portion of the injected power is absorbed in the plasma before the waves reach the sniffer probe at the 9.5-L port. We should mention that the signal level at the 2-O sniffer probe for the case of $X$-mode wave injection to overdense plasmas is as low as that for the case of the $O$-mode wave injection. We injected left handed and right handed circularly polarized waves instead of the pure $O$-mode and $X$-mode waves. Therefore the injected $X$-mode waves had $O$-mode wave components to some extent and the electron temperature rose a little as shown in Fig. 3(d) because of the absorption of the excited EBWs. Moreover, the cutoff point of the $X$-mode wave is different from that of the $O$-mode wave, thus the large portion of the reflected wave may not come back directly around the 2-O port. These might be the reasons why the stray radiation level for the case of $X$-mode wave injection is as low as that in the case of $O$-mode wave injection into overdense plasma.

\section{CONCLUSION}

ECH by EBWs excited via $O-X-B$ mode conversion has been demonstrated in LHD. The level of stray radiation signal picked up near the injection antenna becomes low when EBWs are successfully excited. It is very helpful just to compare the signal level of the stray radiation to confirm the power absorption without precise analysis. Even if the superimposed power is very low compared to the total heating power, less than $5 \%$, we can judge the effectiveness of superimposed power injection.

The authors are grateful to the technical staff of the LHD group for their helpful support. This work was supported by NIFS code of NIFSO5ULRR501, 502, 503.

${ }^{1}$ F. Gandini et al., Fusion Eng. Des. 56-57, 975 (2001).

${ }^{2}$ J. Preinhaelter and V. Kopecky, J. Plasma Phys. 10, 1 (1973).

${ }^{3}$ E. Mjølhus, J. Plasma Phys. 31, 7 (1984).

${ }^{4}$ H. P. Laqua, V. Erckmann, H. J. Hartfu $\beta$, H. Laqua, and W7-AS Team ECRH Group, Phys. Rev. Lett. 78, 3467 (1997).

${ }^{5}$ K. Nagasaki and N. Yanagi, Plasma Phys. Controlled Fusion 44, 409 (2002).

${ }^{6}$ H. Igami, H. Tanaka, and T. Maekawa, Plasma Phys. Controlled Fusion 48, 573 (2006).

${ }^{7}$ A. Müeck et al., 32nd European Physical Society Conference on Plasma Physics and Controlled Fusion combined with the Eight International Workshop on Fast Ignition of Fusion Targets, Tarragona, 27 June-1 July 2005 (European Physical Society, Geneva, 2005). 